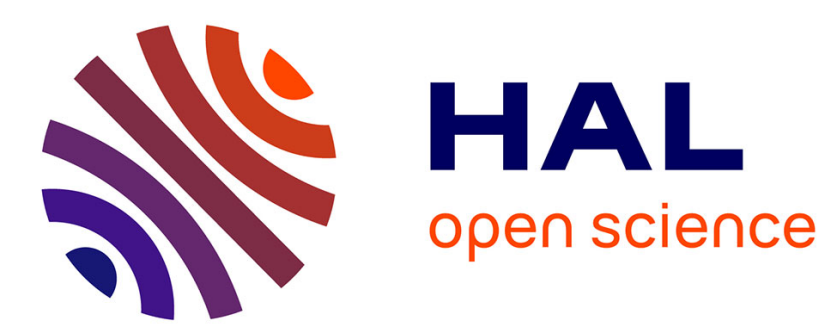

\title{
Une singularité dans l'aide à la décision: le cas de la Liaison 16
}

\author{
Cécile Godé, Valérie Hauch, Mélanie Lasou, Jean-Fabrice Lebraty
}

\section{To cite this version:}

Cécile Godé, Valérie Hauch, Mélanie Lasou, Jean-Fabrice Lebraty. Une singularité dans l'aide à la décision: le cas de la Liaison 16. Systèmes d'Information et Management, 2012, 17 (2), pp.9-38. halshs-00728493

\section{HAL Id: halshs-00728493 \\ https://shs.hal.science/halshs-00728493}

Submitted on 6 Sep 2012

HAL is a multi-disciplinary open access archive for the deposit and dissemination of scientific research documents, whether they are published or not. The documents may come from teaching and research institutions in France or abroad, or from public or private research centers.
L'archive ouverte pluridisciplinaire HAL, est destinée au dépôt et à la diffusion de documents scientifiques de niveau recherche, publiés ou non, émanant des établissements d'enseignement et de recherche français ou étrangers, des laboratoires publics ou privés. 


\title{
Une singularité dans l'aide à la décision : le cas de la Liaison 16
}

\author{
Cécile GODE*, Valérie HAUCH**, Mélanie LASOU*** \& Jean-Fabrice \\ LEBRATY****
}

\author{
* Centre de Recherche de l'Armée de l'Air (CReA) \\ **Université de Nice Sophia Antipolis \& Laboratoire GREDEG \\ ***Ecole Militaire de l'Armée de l'air \\ ****Université de Lyon3 \& Laboratoire MAGELLAN
}

\begin{abstract}
Résumé
Cet article se penche sur un type particulier de Système d'Aide à la Décision (SAD), les systèmes d'aide à la décision centrés réseaux, et pose la question de ses effets sur la créativité des décideurs lorsqu'ils sont confrontés à des situations extrêmes. Une étude de cas exploratoire, s'intéressant aux potentialités créatives d'un SAD militaire centré réseaux, est développée. Il s'agit d'étudier les effets d'une liaison de données tactiques appelée Liaison 16 sur la créativité de ses usagers, en l'occurrence des pilotes de chasse sur Rafale. Le corpus des données de terrain a été construit par triangulation de 33 entretiens individuels, d'observations non participatives ainsi que d'archives et de rapports officiels. L'analyse des résultats suggère que ce système d'aide à la décision favorise la créativité, bien qu'il n'ait pas été conçu en ce sens. L'émergence de créativité résulte de la combinaison entre les caractéristiques de flexibilité du système tout en respectant des règles strictes, l'intégration de ce système dans un processus organisationnel et les capacités intrinsèques des utilisateurs.
\end{abstract}

Mots clefs

Créativité, Situation extrême de gestion, Système d'Aide à la Décision, Décision en situation.

\section{Decision Support Oddness: The case of Link 16}

\begin{abstract}
This article aims at studying net-centric decision support systems. The main research question examines the effect of such systems on decision maker creativity in extreme situation. We perform an exploratory case study in the French Air Force context. The system, currently used on operational theaters, is known as Link 16. Based upon triangulation of 33 interviews, shadowing observations as well as the analysis of doctrinal documentations and reports from the field, we show that Link 16 increases decisional creativity. Although the tactical system has not been designed to support creativity, it leads to occurrence of creativity in decision making thanks to (1) flexibility in the interface in accordance with strict rules, (2) integration into organizational process and (3) users' capabilities.
\end{abstract}

Key words: Creativity, Decision support system, Extreme situation, Decision in Natural settings. 


\section{INTRODUCTION}

La question du rôle joué par les Technologies de l'Information et de la Communication sur les capacités créatives des usagers n'a été posée que relativement tardivement par la littérature en management des systèmes d'information. Mis à part quelques articles publiés à la fin des années 80 (1987, Applegate et al., 1988), il faut réellement attendre le milieu des années 90 pour bénéficier d'analyses rigoureuses traitant spécifiquement du lien entre créativité et système d'information (Couger et al., 1993, Abraham et Boone, 1994, Massetti, 1996, Wierenga et van Bruggen, 1998). Ces contributions majeures ont encouragé la publication de nombreux travaux autour de la créativité, pour certains parus dans les meilleures revues de la discipline (Cooper, 2000, Forgionne et Newman, 2007, Sung et Choi, 2012).

Depuis, la recherche dans le domaine a pris trois orientations majeures (Cooper, 2000) : la première concerne l'identification des démarches, modèles et outils de travail susceptibles de favoriser la créativité lors du développement des technologies. Cette perspective permet par exemple d'aborder la question des effets du climat organisationnel sur les capacités créatives des concepteurs de technologies. La deuxième orientation s'intéresse à la façon de mettre en œuvre et diffuser ces modèles et outils de créativité aux membres de l'organisation (Galletta et al., 1992). Il s'agit alors de décrire les interventions managériales les plus à même de favoriser l'adoption de ces techniques. Ces deux premières perspectives questionnent la créativité lors de la conception du design de la technologie et de son implémentation. Enfin, la troisième orientation de recherche interroge la valeur créative des systèmes d'aide à la décision (SAD) individuels et de groupe, cherchant à conceptualiser ce que les auteurs appellent des systèmes d'aide à la créativité. Comme le précisent Wierenga et Bruggen (1998), les systèmes d'aide à la créativité (SAC) sont des SAD qui révèlent les potentialités créatives des individus dans l'usage : "les systèmes d'aide à la décision [...] ayant pour objectif d'aider les décideurs, peuvent générer de nouvelles formes d'action et produire des idées innovantes et utiles » (p. 81).

Cet article s'inscrit dans cette troisième et plus récente perspective qui considère que les SAD sont en mesure de favoriser l'émergence et la mise en œuvre de nouvelles pratiques de décision. Ils sont identifiés comme des SAC a posteriori, lorsque leurs usages conduisent les individus à valoriser leurs potentialités créatives. Ainsi, à travers les usages quotidiens et situés du SAD (Orlikowski, 2002), les acteurs développent des pratiques de décision adaptées aux situations auxquelles ils sont confrontés, contribuant à l'émergence de processus de créativité. Plus précisément, nous adoptons une approche socio-matérielle. Comme le souligne Orlikowski (2007), les caractéristiques techniques de l'outil technologique, sa configuration, ses modes de programmation et le contexte au sein duquel il est introduit sont inextricablement liés aux effets sociaux de ses usages. Dans ce cadre, la matérialité de l'artefact technologique cristallise un ensemble de ressources tangibles offrant aux usagers la possibilité de faire évoluer leurs façons de faire et d'innover dans leurs modes d'utilisation (Leonardi et Barley, 2008).

L'article s'intéresse à un type particulier de SAD dont les effets créatifs n'ont encore jamais été considérés : les systèmes d'aide à la décision centrés réseaux. Ces systèmes structurés en réseaux procurent aux décideurs une représentation du problème qu'ils ont à résoudre à partir d'une visualisation des éléments d'intérêt associés au problème et des liens entre ces éléments (Godé et Lebraty, 2013). Comme le soulignent Kwon et Watts (2006), il est essentiel de prendre en compte les facteurs environnementaux externes afin d'appréhender finement les apports des systèmes d'information et, pour ce qui nous intéresse, le lien entre créativité et système d'aide à la décision. Dans cet article, nous choisissons d'étudier ce lien lorsque les SAD centrés réseaux sont utilisés en situations extrêmes de gestion, c'est-à-dire lorsque les usagers évoluent dans un environnement marqué à la fois par l'évolutivité, l'incertitude et le risque (Bouty et al., 2011). Nous considérons en effet que les effets paroxysmiques associés à 
de telles situations permettent d'observer la façon dont les acteurs décident sous contraintes et de révéler des usages difficilement discernables lors de situations de gestion plus classiques.

La question de recherche soulevée dans cet article est la suivante : quels sont les effets d'un système d'aide à la décision de type centré réseaux sur la créativité lorsque les décideurs sont confrontés à des situations extrêmes?

Afin d'y répondre, l'article se propose de décrire les effets d'un SAD centré réseaux militaire, la Liaison 16, sur la créativité des pilotes lorsqu'ils réalisent des missions opérationnelles. Deux des auteurs de cet article ont été impliqués dans des recherches visant à répondre à la question des effets de la Liaison 16 sur la prise de décision et la créativité des pilotes usagers. Ces recherches ont représenté une opportunité rare d'explorer un terrain encore méconnu en Sciences de Gestion, et en Management des Systèmes d'Information en particulier. Il se révèle bien adapté à notre problématique dans la mesure où il permet d'une part d'étudier un SAD centré réseaux, courant dans le milieu militaire et beaucoup moins dans le milieu civil, et, d'autre part, d'analyser les effets de ce système sur la créativité en opérations militaires, c'est-à-dire en situations extrêmes.

L'article s'organise en quatre sections : la première définit les concepts clés et développe les fondements théoriques retenus. La deuxième section présente le terrain d'étude ainsi que la méthodologie adoptée. La troisième développe les résultats de/l'étude de cas. Enfin, la quatrième section discute les résultats de terrain afin d'approfondir la notion de créativité et de mettre en lumière les effets des systèmes d'aide à la décision centrés réseaux dans ce domaine.

\section{CADRE THEORIQUE}

L'objet de cette section est de proposer une revue de littérature des concepts de créativité et de situation extrême à l'aune des systèmes d'aide à la décision.

\section{I.1 Créativité et système d'aide à la décision}

Généralement, la créativité est considérée comme la production d'idées, de biens, de services ou de processus à la fois nouveaux et porteurs de valeur pour l'organisation (Amabile, 1988, Woodman et al., 1993). Pour autant, le concept, tel qu'il est traité par la littérature reste «une boite noire monolithique " attendant encore d'être explorée (Seidel et al., 2010). La créativité apparaît ainsi comme une notion polymorphe et englobant de multiples réalités : elle peut se référer tour à tour à des capacités (individuelles ou collectives), à un processus cognitif, à son résultat et à son environnement. Au regard de ces différentes caractéristiques, nous définissons la créativité comme un processus individuel et/ou collectif d'évolution de la décision visant une meilleure adéquation entre les choix d'actions opérés et l'environnement. Nous retrouvons ainsi les quatre dimensions de la créativité communément identifiées depuis Rhodes (1961): personne/groupe, processus de création, produit/service et environnement, que nous nous proposons maintenant de détailler.

La créativité des acteurs dépend tout d'abord de traits de caractère, d'aptitudes particulières dont Torrance (1974) a d'ailleurs proposé une célèbre série de mesures autour de l'aisance dans la génération d'idées, de la flexibilité de pensée et de l'originalité dans la sélection d'associations d'idées. Plus tard, Csikszentmihalyi (1996), en étudiant le parcours de 91 scientifiques et artistes particulièrement créatifs, a pu identifier des traits de caractère communs : la combinaison de l'imagination et du sens des réalités ainsi que la capacité de persévérer dans l'étude d'un sujet qui va animer et motiver les acteurs, les plaçant dans un état de «flux », c'est-à-dire une concentration totale de leur énergie sur leur travail. Cette attention permanente, relevant de la même dynamique que l'apprentissage expérientiel, va permettre aux acteurs de mettre à profit chaque élément de vécu pour construire leurs 
nouvelles idées ou leur création. D'un point de vue collectif, la créativité est envisagée à travers la dynamique des échanges et de partage des connaissances à l'intérieur d'équipes. Les auteurs s'intéressent, dès lors, au niveau d'expertise des membres du groupe (Amabile, 1997, Gobet, 2002) ainsi qu'à la richesse des interactions en son sein (Mascitelli, 2000).

La créativité relève, ensuite, d'un processus cognitif complexe lié aux connaissances des individus, aux informations disponibles et à leur combinaison (Mednick, 1962). Depuis les travaux fondateurs de Wallas (1926), on identifie généralement quatre étapes présidant à ce processus de créativité : la phase de préparation, au cours de laquelle les informations internes (expériences, connaissances) et les informations externes (données de l'environnement) sont mobilisées et recueillies; la phase d'incubation consistant en la combinaison sous la forme d'associations d'idées des éléments mentaux mis en œuvre précédemment; la phase d'illumination, durant laquelle les idées affluent au niveau du conscient; la phase de vérification, qui voit les nouvelles idées mises à l'épreuve de la réalité et ainsi sélectionnées. Dans cette vision de la créativité, le raisonnement par analogie, fondé sur la confrontation de sources variées d'inspiration est au centre du processus d'émergence d'idées nouvelles, comme le souligne Bonnardel (2000) dans son étude sur le travail des designers.

La créativité peut également être considérée comme un résultat "situé » et recherché par un individu ou un collectif afin d'accroître sa performance. Dans ce cadre, une solution créative repose sur un processus combinatoire non encore exploré par les acteurs et qui procure de la valeur à la fois au niveau du collectif (l'équipe, par exemple) et de l'organisation (Couger et al., 1993, Fellers et Bostrom, 1993, Seidel et al., 2010).

Enfin, la créativité est fortement liée à l'environnement dans lequel évoluent les individus, celui-ci incluant les ressources mises à disposition, le degré d'autonomie, la composition des équipes et la communication entre leurs membres (Amabile, 1988). De nombreuses études ont ainsi été menées sur des éléments variés de cet environnement de travail : dimension collaborative (Mascitelli, 2000); environnement physique et organisation de l'espace (Kristensen, 2004); pression temporelle (Amabile et al., 2002) ; système d'information (Couger et al., 1993, Bonnardel et Marmèche, 2005, Seidel et al., 2010).

Dans cette dernière approche, depuis une vingtaine d'années, des chercheurs en management des systèmes d'information interrogent la contribution des SAD à la créativité s'exprimant au niveau de la décision, qu'elle soit individuelle ou collective (Forgionne et Newman, 2007).

Rappelons tout d'abord qu'un SAD vise à fournir une assistance à un décideur par la possibilité de gérer des données et des informations au travers d'une interface conviviale (Sprague et Watson, 1996, Alter, 2004). Il existe, selon Power et Sharda (2007), cinq types de SAD, mais cette taxinomie ne diffère globalement pas de la dichotomie fondamentale de Alter (1977) :

- des systèmes permettant une manipulation de données et laissant ainsi au décideur la capacité de générer, par lui-même, de l'information à partir des données ainsi agencées ;

- des systèmes intégrant un modèle (de simulation, par exemple) et fournissant des options au décideur qui exercera ensuite son choix.

Très vite est apparue l'idée selon laquelle ces systèmes d'aide pourraient être à la source d'idées nouvelles. Souvent qualifiés de systèmes d'aide à la créativité (SAC) - ou Creativity Support Systems - ces SAD permettent à leurs usagers de prendre de la hauteur au regard des problèmes qu'ils ont à traiter, leur permettant ainsi d'explorer des points de vue nouveaux et de les conduire à mettre en œuvre des décisions originales, adaptées aux situations. Il s'agit, par exemple, d'un système présentant des données issues de diverses sources d'inspiration, proches ou lointaines par rapport au domaine d'expertise des décideurs (Bonnardel et Marmèche, 2005), ou encore d'un système qui, à partir d'une base de connaissances relatives au problème à traiter, organise et structure les idées et les concepts puis simule un ensemble 
de solutions parmi lesquelles l'usager opérera un choix. Pour Massetti (1996), il est possible de mesurer la valeur créative de tels systèmes en comptabilisant la production de biens ou services nouveaux et/ou l'émergence de processus décisionnels originaux dont ils auraient favorisé le développement. Selon Wierenga et Bruggen (1998), les SAC sont des SAD qui, à travers les usages des individus, se révèlent susceptibles de favoriser l'émergence d'une dynamique créative.

Dans cet article, nous nous concentrons sur un système d'aide à la décision peu étudié par la littérature, les SAD centrés réseaux. La section suivante définit ce type particulier d'outils et explicite en quoi il apparait adapté à la prise de décision en situations extrêmes de gestion.

\section{I.2 Les systèmes d'aide à la décision centrés réseaux : des SAD adaptés à la prise de décision en situations extrêmes}

Comme nous l'avons brièvement énoncé en introduction, les SAD centrés réseaux, sont des $\mathrm{SAD}$ ayant pour objectif de fournir des données (c'est-à-dire des éléments indépendants de l'utilisateur que l'on pourrait qualifier de faits objectifs). Ces données sont agencées sur l'interface utilisateur selon les caractéristiques du cadre d'emploi (amis, ennemis, neutres, éléments topographiques, notamment). L'utilisateur générera alors de l'information (c'est-àdire qu'il apportera du sens aux données) en manipulant ces données. Cette information constituera alors le fondement de son processus décisionnel. En ce sens et sélon la définition de Alter (1977), ce sont des SAD orientés données. Cependant, il convient d'ajouter une caractéristique essentielle: ces systèmes sont conçus pour gérer des opérations centrées réseaux, c'est-à-dire, des opérations pour lesquelles la compréhension des interactions entre différents acteurs hétérogènes est essentielle (Benssam et al., 2007). Ces systèmes sont majoritairement employés dans le domaine militaire, aussi, la recherche académique en management ne les a-t-elle que peu explorés. Nous pouvons cependant définir les SAD centrés réseaux de la façon suivante : ce sont des "systèmes d'aide à la décision permettant la visualisation de l'ensemble des acteurs d'une situation et possédant au moins les trois fonctions suivantes : prise en compte de données géographiques, manipulation des données sous la forme de calques (chaque calque regroupant un type d'acteur) et drill-down (possibilité de zoomer sur les caractéristiques d'un élément particulier et de remonter ensuite vers une vue globale) ».

Les systèmes d'aide à la décision centrés réseaux permettent de collecter une quantité importante de données à partir de toutes les sources disponibles et de les transmettre vers l'ensemble des nœuds du réseau. Reposant sur un réseau structuré, ils opèrent une collecte et une diffusion régulière et rapprochée des données disponibles. Celles-ci sont ainsi actualisées (ou rafraîchies) en quasi temps réel. Les SAD centrés réseaux récupèrent, organisent et analysent ces données afín de fournir aux décideurs une visualisation et une représentation appropriée du problème qu'ils doivent traiter. La visualisation fait ici référence au processus de conversion de l'information non visuelle (textuelle, numérique ou sonore) sous forme graphique (Meyer, 1997). La littérature souligne en effet que les usagers de systèmes d'aide à la décision s'approprient et traitent plus efficacement l'information lorsque celle-ci est présentée sous forme visuelle (Huang et al., 2006), en particulier lorsque les décideurs évoluent en situation d'incertitude (Comes et al., 2011). La visualisation des informations contribue à produire une représentation : il s'agit de présenter graphiquement les éléments situationnels ainsi que les liens qui les unissent en mobilisant des règles de modélisation spécifiques (Fuller et al., 2010). Les systèmes d'aide à la décision centrés réseaux sont ainsi en mesure de procurer aux décideurs une représentation du problème qu'ils ont à résoudre à partir (1) d'une visualisation des éléments d'intérêt associés au problème et (2) des liens entre ces éléments. La représentation est considérée comme appropriée lorsqu'elle répond aux besoins des décideurs, c'est-à-dire lorsque la représentation du problème mentalement 
élaborée par le décideur correspond à celle produite par le système (Shaft et Vessey, 2006, Baker et al., 2009).

Cet article propose d'étudier l'usage des SAD centrés réseaux en situations extrêmes de gestion. Afin de préciser le caractère extrême d'une situation, nous nous fondons sur la définition d'une «situation de gestion» proposée par Girin (1990) lorsqu'il évoque "des participants [...] réunis et devant accomplir, dans un temps déterminé, une action collective conduisant à un résultat soumis à un jugement externe » (p. 142). Comme le précisent Journé et Raulet-Croset (2008), cette définition met l'accent sur les dimensions collective, spatiale et temporelle de la situation, tout en insistant sur la question du résultat et de la performance. Dans cet article, nous considérons qu'une situation de gestion devient extrême lorsqu'elle se déroule dans un environnement marqué à la fois par l'évolutivité, l'incertitude et le risque (Bouty et al., 2012). La notion d'évolutivité renvoie à celle de volatilité, qui souligne les aspects rapides, dynamiques et discontinus des changements auxquels les individus font face (Bourgeois et Eisenhardt, 1989, Wirtz et al., 2007, McCarthy et al., 2010), tout en insistant sur l'existence de décalages dynamiques par rapport à un mode de fonctionnement antérieur. Le critère d'incertitude suggère qu'une situation peut être plus ou moins attendue - référence faite à sa probabilité d'occurrence - et/ou plus ou moins anticipable - selon le niveau de prévisibilité du moment et des modalités de la situation (Bouty et al., 2011). Enfin, le risque évoque la possibilité qu'un évènement non souhaité survienne et cause des dommages physiques, symboliques et/ou matériels plus ou moins importants à l'organisation et/ou à ses acteurs.

Les situations extrêmes de gestion ne renvoient pas forcément à la crise. Selon Roux-Dufort (2004), la crise s'inscrit "en dehors des cadres opératoires typiques de l'organisation et bouleverse son cadre de référence » (p. 15). Plus précisément, en référence à l'approche par contraste entre l'accident classique, l'évènement majeur et la crise proposée par Lagadec (1991), cette dernière correspond à (1) une situation d'urgence qui déborde les capacités des acteurs et des structures, (2) une menace de désagrégation du système et de l'univers de référence (pp. 57-59). En situations extrêmes de gestion, le décideur ne se retrouve pas forcément face au «trou noir » dont parle Lagadec, à savoir saturé et totalement dépassé par l'évènement. La nature "extrême » de la situation doit être envisagée en termes de degré. Plus exactement, les acteurs élaborent des processus d'action à partir de situations « prévisibles » ou « routinières », tout en prenant en compte la probabilité d'être confrontés à l' « imprévu » ou au " non routinier». Un évènement est prévisible lorsqu'il correspond à une attente. Il s'inscrit dans des modèles d'action standard, élaborés en amont par l'acteur. A l'inverse, un évènement imprévisible implique la remise en cause, partielle ou totale, de ces standards. Les individus font alors face à une réalité inattendue, dont la probabilité d'occurrence est faible. Le fait que la situation soit imprévisible ne signifie pas qu'elle soit forcément inconnue des acteurs. Ils ont déjà pu avoir à en gérer de similaires, mais n'avaient pas prévu de le faire à ce moment-là de la situation de travail. Une situation imprévisible est donc source de surprise pour les acteurs, qui doivent agir sans modèles prédéfinis pour mener à bien leurs objectifs initiaux. Pour autant, elle ne renvoie pas systématiquement à la crise dans la mesure où les décideurs sont sensibilisés à ces potentiels basculements entre la routine et l'inattendu ; ils bénéficient d'une formation dédiée et les structures et outils de décision mis à leur disposition sont adaptés à ce contexte d'action particulier. L'intérêt d'étudier l'activité managériale, et plus particulièrement la prise de décision en situations extrêmes de gestion réside dans les effets paroxysmiques qui y sont associés. Elles permettent en effet d'observer la façon dont les acteurs se comportent sous de fortes contraintes et de révéler des pratiques et usages difficilement discernables lors de situations de gestion plus classiques. Ainsi, "les situations extrêmes de gestion constituent un laboratoire naturel (au sens de non 
expérimental) et privilégié d'étude » (Bouty et al., 2011p.390) pour approfondir notre compréhension et notre connaissance du fonctionnement de l'organisation.

En situation extrême de gestion, plus qu'ailleurs, la distinction entre expert et novice sera déterminante (Randel et Pugh, 1996, Hung, 2003). Dans cet article, nous nous focalisons sur des décideurs experts; aussi, le modèle IDS de Simon (1997) conduisant à examiner un ensemble d'options ne convient que partiellement (Kahneman et Klein, 2009). Nous abordons plutôt la décision à partir du modèle " naturaliste » développé par Klein (1998) qui s'applique spécifiquement à notre contexte. Ce modèle repose sur un processus de première reconnaissance mis en œuvre par des experts qui, loin de comparer plusieurs options entre elles, en évaluent une seule et l'appliquent si elle leur paraît adaptée à la situation. Selon l'approche naturaliste, l'attention doit porter sur la situation décisionnelle ; c'est une prise en compte holistique du contexte, "un peu comme celle du joueur d'échec jetant un oil sur une partie en cours, opposant deux joueurs ; une association se produit alors entre l'image qu'il vient d'enregistrer et une référence mentale en stock dans son cerveau, comprenant à la fois contexte et modus operandi $»$ (Lebraty et Lebraty, 2010 p.140). Cette approche possède une implication forte en termes d'aide à la décision. Celle-ci doit porter sur le début du processus décisionnel et non sur la fin. En d'autres termes, il est essentiel que le décideur comprenne la situation dans laquelle il se trouve plutôt que de lui fournir un ensemble d'options parmi lesquelles il devrait choisir. C'est alors tout l'intérêt présenté par des SAD centrés réseaux. En effet, ces systèmes proposent une vision globale d'une situation et, en permettant une manipulation des données, laissent l'utilisateur développer sa compréhension de la situation. Il ne s'agira pas ensuite de proposer des options, car les décideurs étant experts, ils n'en ont pas besoin : une fois la situation comprise, ils savent comment agir. Par leurs caractéristiques, les SAD centrés réseaux favorisent la prise de conscience de la situation par le décideur (Endsley et Garland, 2000). En offrant la possibilité d'acquérir cette conscience, ils supportent efficacement des experts confrontés à des processus de décision extrêmes.

Arrivé au terme de cette revue de littérature, il apparaît légitime de se demander où peut se situer la créativité dans des processus décisionnels naturalistes épaulés par des SAD centrés réseaux. La réponse à cette question s'exprime en deux temps. Premièrement, nous lions créativité et décision naturaliste. Nous estimons que la créativité constitue la mise en pratique d'un mode d'action inédit pour le décideur par rapport à une situation qu'il a reconnue comme typique. Un exemple illustre parfaitement cette définition : quand, en janvier 2009, face à une panne de moteur, le commandant de bord Chesley Sullenberger a posé son A320 sur l'Hudson, il a fait preuve de créativité. Deuxièmement, nous nous reposons sur le fait que le $\mathrm{SAD}$ centré réseaux joue un rôle particulier : celui de constituer le moyen principal du décideur pour prendre conscience de la situation qu'il doit traiter.

\section{CONTEXTE DE LA RECHERCHE ET MÉTHODOLOGIE}

Dans ce point, nous présenterons les caractéristiques générales du système et de notre terrain, ainsi que la méthodologie mise en œuvre.

\section{II.1 La Liaison 16}

Depuis 2006, la France équipe progressivement ses forces aériennes de la technologie JTIDS (Joint Tactical Information Distribution System) américaine. Egalement appelé «Liaison $16 »$, ce système a été implémenté dans l'avion de chasse polyvalent Rafale ${ }^{1}$, l'avion de

\footnotetext{
${ }^{1}$ La polyvalence fait ici référence à la capacité de l'appareil à réaliser l'ensemble des missions tactiques, à savoir la Défense aérienne (surveillance et protection de l'espace aérien), le bombardement (stratégique et tactique) et la reconnaissance (reconnaissance photographique et surveillance de zones prédéfinies), ainsi que les missions stratégiques, c'est-à-dire le bombardement nucléaire.
} 
commandement et de contrôle Awacs (Airborne Warning and Control System) ${ }^{2}$ et plusieurs Mirage $2000 \mathrm{D}^{3}$.

Reposant sur une structure réticulée hautement sécurisée (système de cryptage), la Liaison 16 soutient l'échange de données tactiques essentielles à la coordination des unités et des platesformes (aériennes, terrestres et maritimes) militaires. Les données recueillies par les différents capteurs disséminés sur la zone de combat (humains et technologiques) sont fusionnées par la Liaison 16 qui élabore une "image » cohérente et actualisée de la situation de terrain. Cette représentation tactique est diffusée vers les différents acteurs impliqués dans 1'opération, tels que le haut commandement ou Reachback (le Pentagone aux Etats-Unis ou le Centre de Planification et de Commandant des Opérations (CPCO) en France par exemple), les centres de commandement tactiques localisés sur théâtre (c'est-à-dire la zone terrestre, aérienne et/ou maritime où se déroulent les opérations militaires) ou encore les acteurs et plates-formes impliqués dans les missions particulières à réaliser. Comme le précise un officier d'Etatmajor : "l'information tactique peut être transmise grâce à la liaison de données qui permet de communiquer sous forme numérique des données vers le sol, vers un autre vecteur comme l'Awacs ou encore vers d'autres Rafale ». Les acteurs et plates-formes peuvent à la fois être des usagers de la Liaison 16 et des «capteurs », dans la mesure où les données récoltées lors de leurs missions contribuent à l'actualisation de la représentation tactique. Cet article se concentre sur ce dernier type d'acteurs, et plus particulièrement sur les pilotes de Rafale.

Les données tactiques échangées par les pilotes grâce à la Liaison 16 concernent par exemple la position des forces amies et ennemies, le niveau de carburant, l'armement disponible, les conditions météorologiques, les coordonnées de cibles, les terrains de déroutement et la position des avions ravitailleurs (figure 1). La Liaison 16 leur procure également des capacités de communication via un langage commun (les données de série-J) facilitant leur coordination en situation. L'ensemble de ces services permet aux usagers d'améliorer leur «Situational Awareness - $S A$ » (Endsley et Garland, 2000). Comme ils le précisent lors des interviews, la Liaison 16 leur permet d'obtenir simultanément: (1) la perception des éléments environnementaux dans un volume de temps et d'espace : "la première chose que l'on échange, c'est la position de chaque participant et des forces ennemies parce que lorsqu'on sait où est chaque acteur et à quel moment, on se coordonne plus facilement » (un pilote de Rafale) (2) la compréhension de leur signification : «car avec la Liaison 16, on va savoir en permanence où se trouvent les amis, les ennemis et on obtient une vision globale de la situation » (un pilote de Rafale) (3) la capacité d'anticipation de leur évolution «par exemple, la Liaison 16 elle nous donne les tankers [les ravitailleurs], ce qui nous permet de voir graphiquement que dans notre zone, le tanker ne va pas tarder à rentrer car il ravitaille déjà une patrouille, donc nous, on s'adapte. On a toujours un temps d'avance en fait " (un navigateur de Rafale).

\footnotetext{
${ }^{2}$ L'AWACS réalise des missions de détection et de contrôle aériens en appui aux objectifs des forces sur théâtre, tels que le contrôle air-air, le contrôle air-sol, la gestion de l'espace aérien.

${ }^{3}$ Appareils principalement dédiés aux missions de bombardement tactique, tel que l'appui feu rapproché (close air support).
} 


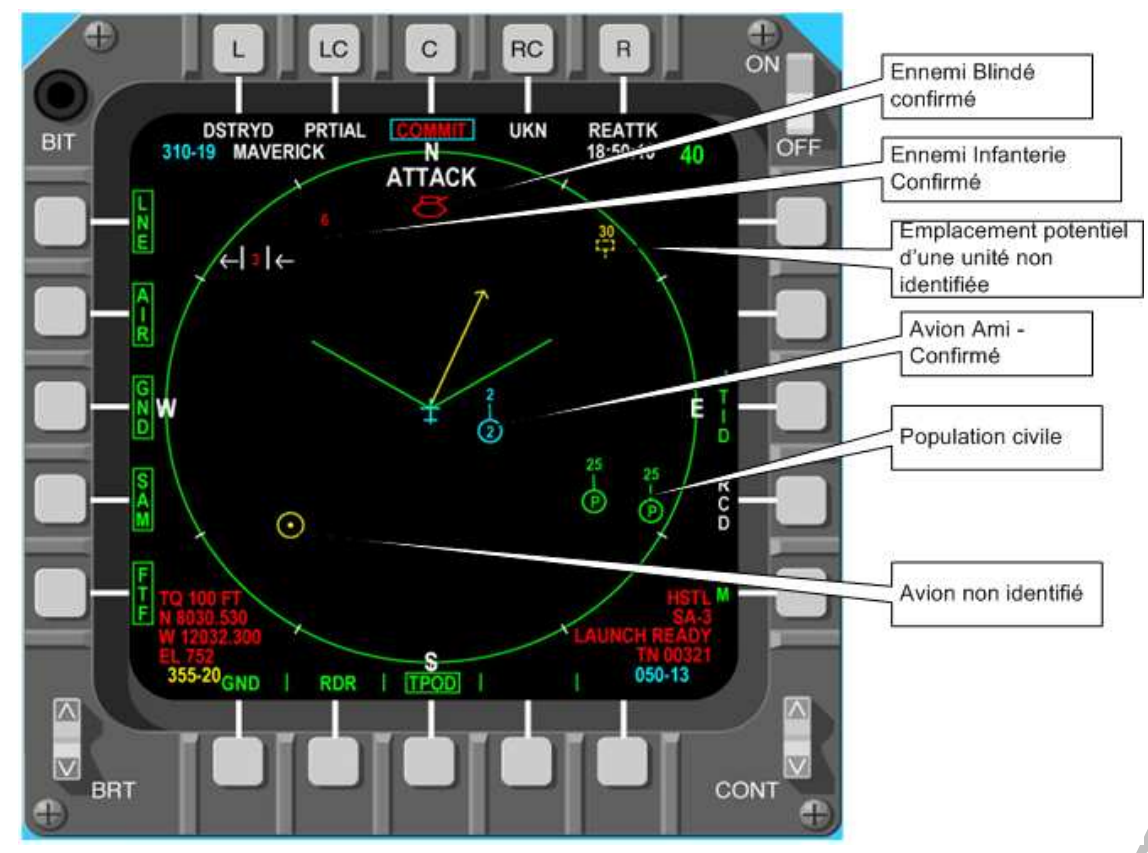

Figure 1 : Exemple de représentation tactique fournie par la Liaison 16 à un pilote de Rafale

La Liaison 16 a été utilisée en Lybie, elle soutient actuellement les missions de bombardement appelées appui feu rapproché (ou Close Air Support) sur le théâtre afghan ainsi que les missions permanentes d'alerte pour la défense aérienne (la posture permanente de sécurité) réalisées au-dessus du territoire français. Le système est donc mis en œuvre au sein de situations que l'on peut qualifier d'extrêmes (Bouty et al., 2012) : la situation peut être amenée à changer très rapidement (des conditions météo instables, un problème mécanique), l'incertitude est forte quant à la survenance d'un évènement inattendu (par exemple des forces au sol prises en embuscade) et le niveau de risque (vital notamment) est élevé. Les pilotes usagés de la Liaison 16 et engagés en Lybie et en Afghanistan sont pour la plupart issus de l'escadron 1/7 Provence de la base de Saint Dizier. Cet escadron est principalement constitué de pilotes expérimentés, pour nombreux d'entre eux qualifiés chef de patrouille (la qualification la plus élevée sur avion de chasse) et accumulant plusieurs milliers d'heures de vol sur avion de chasse. Ces pilotes, experts dans leur domaine, ont également la responsabilité de former les jeunes pilotes en instruction arrivant à l'escadron.

Après quatre années d'utilisation, les retours d'expérience des pilotes de Rafale et des contrôleurs Awacs vont tous dans le même sens : la Liaison 16 offre une sérénité et un confort sans précédent, à la fois en termes d'appréhension de l'environnement d'action que de réactivité face aux menaces. En guise d'illustration, un pilote nous explique : «La Liaison 16 permet d'obtenir une information rafraîchie [actualisée] par les différents capteurs. Il en résulte un confort accru car la position des pistes amies et ennemies est connue et la situation tactique est facilement et instantanément partagée entre les membres du dispositif ». Un autre résume: "la clé de la décision réside dans la bonne représentation de la situation opérationnelle, et c'est ce que nous procure la Liaison $16 »$.

Ces constatations générales conduisent naturellement à interroger les implications créatives d'un tel système. Quels sont les effets de la Liaison 16 sur la créativité des pilotes usagers ?

\section{II.2 Une journée type en escadron de chasse Rafale}

La journée de travail type d'un escadron d'avions de chasse français (Godé et Barbaroux, 2012) commence toujours par un briefing général d'une trentaine de minutes réunissant l'ensemble des pilotes et des navigateurs ayant un ou plusieurs vols prévus. Les pilotes de chasse de l'Armée de l'air française réalisent une moyenne annuelle de 180 heures de vol 
(objectif inscrit dans la Loi de Programmation Militaire 2009-2014). Les vols ont une durée variable, au regard des missions effectuées ainsi que de leur nature, opérationnelle ou d'entraînement. Durant ce «briefing du matin » sont notamment présentés les conditions météorologiques du jour, l'état des pistes, la «question du jour » (un pilote ou un navigateur en instruction expose ses connaissances sur un problème de sécurité des vols) et les éventuelles nouvelles règles et procédures à respecter (présentées par le chef d'escadron). Ce moment représente l'opportunité pour les personnels présents de discuter de leurs expériences relatives aux problèmes et risques exposés. Les jeunes pilotes sont encouragés par leur instructeur à participer à ces échanges relativement informels, en faisant part de leurs propres expériences et en questionnant les plus anciens.

A la suite de ce briefing général, les pilotes de chasse se séparent en patrouilles ${ }^{4}$ afin de participer à un briefing spécifiquement dédié au(x) vol(s) qu'ils devront effectuer. Ce briefing est réalisé juste avant le décollage. Les personnels navigants décrivent toutes les phases du vol, les procédures relatives à la sécurité et terminent par ce qu'ils appellent les what-if. Cette dernière étape pose la question de savoir quoi faire (quelles procédures appliquer) si un problème associé au fonctionnement du système survient en vol, comme une panne moteur par exemple. Bien que les missions soient préparées avec rigueur, les combattants peuvent se retrouver face à des situations qui n'ont pas été briefées. Un pilote explique : " entre ce qui a été planifié et ce qu'on vit en l'air, c'est potentiellement très différent. C'est dans le moment qu'on découvre l'inattendu et il faut savoir s'adapter en temps réel ».

Immédiatement après le vol, les pilotes se retrouvent pour un debriefing de mission très formalisé qui dure entre 40 et 45 minutes. La dimension formelle de l'exercice est directement associée aux procédures de debriefing suivies, standardisées dans un manuel publié par l'OTAN. Plus précisément, il s'agit de reprendre l'ensemble des points évoqués durant le briefing, mais en commençant par la fin en insistant sur la sécurité du vol. La patrouille évalue la qualité de la mission effectuée. Le comportement en vol de chacun des pilotes est scruté avec attention, avec une insistance particulière pour les actions inappropriées (les erreurs), les causes de ces actions et les solutions qu'il aurait été intéressant de mettre en œuvre (Godé et Barbaroux, 2012).

\section{II.3 Méthodologie}

Cet article repose sur une démarche qualitative consistant à élaborer un propos théorique à partir d'une étude de cas extrême. Comme le précise Yin (2003), un cas est extrême lorsqu'il examine une situation concrète rarement - voire jamais - documentée en théorie de l'organisation afin d'enrichir la connaissance scientifique. Nous adoptons une démarche abductive afin de mettre à jour un certain nombre de régularités à partir de l'analyse de nos données de terrain. Comme le précisent Van Maanen et al. (2007), l'approche abductive s'intéresse principalement au «monde de l'empirisme, mais en restant au service de la théorisation [dans la mesure où] l'analyse repose sur un aller-retour continuel entre les données et les concepts» (p. 1149). Nous cherchons donc à exploiter nos données pour explorer et enrichir les perspectives théoriques relatives à la question des effets des SAD sur la créativité.

Recueilli entre septembre 2007 et décembre 2009, le corpus des données de terrain a été construit par triangulation (1) d'entretiens semi structurés (Tableau 1), (2) d'observations non participatives et (3) de documents internes écrits (Eisenhardt, 1989).

\footnotetext{
${ }^{4}$ Une patrouille consiste en deux avions soit monoplace (deux pilotes en tout), soit biplaces (deux équipages soit deux pilotes et deux navigateurs).
} 
(1) Quatorze entretiens ont été conduits avec des pilotes et des navigateurs de Rafale, un entretien avec un pilote d'essais sur Rafale et un dernier avec un chef de mission sur Awacs. Chacune des interviews a été enregistrée puis transcrite. Le guide utilisé était structuré autour des usages de la Liaison 16 en opération et des éléments nouveaux qu'ils ont permis d'introduire en termes décisionnels et tactico-opérationnels. Par ailleurs, douze pilotes de Mirage (non équipés de Liaison 16) ont été interviewés afin de comprendre leurs pratiques de travail sans la Liaison 16 et uniquement avec le système phonique qui équipe aujourd'hui la plupart des avions. Enfin, nous avons rencontré trois officiers supérieurs d'Etat-major en charge de l'élaboration de la doctrine associée à la Liaison 16. Cette démarche de comparaison « avant/après » nous a permis d'appréhender finement les évolutions dont la Liaison 16 a facilité l'introduction.

(2) L'observation non participative de la simulation d'un exercice a permis d'approfondir la compréhension de la façon dont les contrôleurs sur Awacs travaillent avec la Liaison 16. Nous avons également assisté à un briefing du matin à Saint Dizier.

(3) Enfin, l'analyse de nombreux documents internes, de retours d'expérience et de plusieurs rapports d'étude réalisés sous l'égide du Ministère de la Défense français et américain (Dubey et Moricot, 2005, Gonzales et al., 2005, Barbaroux et al., 2008) a également été très utile pour mieux comprendre les évolutions dans les processus de décision et les pratiques de travail. Certains de ces rapports contenaient des extraits de verbatims dont nous nous sommes servis en tant que données secondaires (Heaton, 2004, Kelder, 2005).

\begin{tabular}{|c|c|c|}
\hline Qualité des personnels interviewés & Nombre d'interviewés & $\begin{array}{c}\text { Année de réalisation } \\
\text { de l'interview }\end{array}$ \\
\hline Pilotes de Rafale & 9 & 2008 et 2009 \\
\hline Navigateurs de Rafale & 5 & 2008 et 2009 \\
\hline Pilote d'essais de Rafale & 1 & 2008 \\
\hline $\begin{array}{c}\text { Mission Commander sur Awacs } \\
\text { Pilotes de Mirage-5 (appareil de } \\
\text { Défense aérienne) }\end{array}$ & 1 & 2008 \\
\hline $\begin{array}{c}\text { Pilotes de Mirage 2000D (appareil de } \\
\text { bombardement) }\end{array}$ & 3 & 2007 \\
\hline $\begin{array}{c}\text { Navigateurs de Mirage 2000D } \\
\text { Pilotes de Rafale (données } \\
\text { secondaires) }\end{array}$ & 3 & 2007 \\
\hline Officiers d'Etat-major & 2 & Entre 2006 et 2009 \\
\hline Total & 3 & $\mathbf{3 3}$ \\
\hline
\end{tabular}

Tableau 1 : Panel et effectif des acteurs interviewés

Nous avons construit notre échantillonnage dans une perspective de benchmarking, cherchant à affiner notre compréhension des attributs créatifs de la Liaison 16 au regard des systèmes antérieurs utilisés par les décideurs. Il s'agissait donc d'une part de préciser la notion de 
créativité en milieu opérationnel et, d'autre part, de déterminer les « poches » d'expression de cette créativité (Seidel et al., 2010) suite à l'implémentation de la Liaison 16. Cette démarche de benchmarking est pertinente pour les deux raisons suivantes : tout d'abord, les spécialités d'origine des pilotes de Rafale interviewés sont soit le bombardement (la plupart volant antérieurement sur Mirage 2000D), soit la Défense Aérienne (la plupart volant antérieurement sur Mirage-5). Les procédures de qualifications suivies par les pilotes sont les mêmes que dans n'importe quel escadron de chasse. Dans ce cadre, les compétences individuelles des pilotes de Rafale interviewés sont très proches de celles des pilotes de Défense aérienne et de bombardement, ce d'autant plus que la polyvalence de l'appareil conduira un pilote de Rafale à réaliser l'ensemble des missions de la chasse. Ensuite, les questionnements auxquels sont confrontés les pilotes de Rafale, notamment en termes tactiques, sont les mêmes que lorsqu'ils volaient sur Mirage. La seule différence réside dans les capacités supplémentaires fournies par le Rafale, et par la Liaison 16 en particulier.

Les données récoltées ont été traitées par l'intermédiaire du logiciel NVivo8. Elles concernaient les transcriptions des entretiens, les notes de terrain prises lors des observations in situ consignant le déroulement des tâches exécutées lors de la démonstration au simulateur et certains passages des rapports étudiés. Le traitement des données de terrain a été réalisé par codage ouvert afin de faire émerger les thèmes représentatifs et réguliers au fil de l'analyse.

Comme le précisent Strauss et Corbin (2008), la méthode inductive de codage ouvert permet "de couper, d'examiner, de comparer, de conceptualiser et de catégoriser les données" (p. 61). Elle conduit ainsi le chercheur à révéler un certain nombre de propriétés intrinsèques des catégories découvertes ainsi que le continuum le long duquel elles évoluent (Angot et Milano, 2007). Plus précisément, nous sommes partis du cadre conceptuel afin de déterminer une première liste de codes thématiques. Nous avons donc cherché à identifier les « espaces » de créativité produits par le SAD centré réseaux par rapport à ceux dont les décideurs bénéficiaient antérieurement. La souplesse d'usage du logiciel NVivo8 a permis de révéler de nouveaux thèmes tout au long du traitement du matériel, éprouvant la robustesse de la première liste de codage. Notre arborescence repose sur trois thèmes principaux : (1) les changements induits par l'usage de la Liaison 16, (2) les espaces de créativité et (3) les nouvelles contraintes associées au système (ce dernier thème n'a pas été exploité dans cet article au regard du caractère sensible de certaines données). L'analyse détaillée des données dans les deux premiers thèmes ont permis de révéler (1) des changements au niveau de la boucle de décision, de la communication, des modes de coordination, et (2) les poches principales de créativité avant, pendant et après le vol. L'article est principalement construit autour de ce dernier thème.

Concernant les entretiens réalisés en 2008 avec les usagers de la Liaison 16 (12) et en 2007 avec les pilotes non usagers (12), un compte rendu a chaque fois été produit et transmis aux personnels interviewés. Ces vingt-quatre monographies (près de $73 \%$ des interviews réalisées) ont été l'occasion de confronter nos observations, interprétations et analyses afin de faire émerger des suggestions alternatives et des questionnements qui n'avaient pas été mis à jour jusque-là. Certaines remarques écrites des interviewés ont été importées dans NVivo8 afin d'affiner l'analyse.

A partir du schéma élaboré par Koners et Goffin (Koners et Goffin, 2005 p.338), voici une illustration de la triangulation des données appliquée au thème de la créativité pendant le vol : 


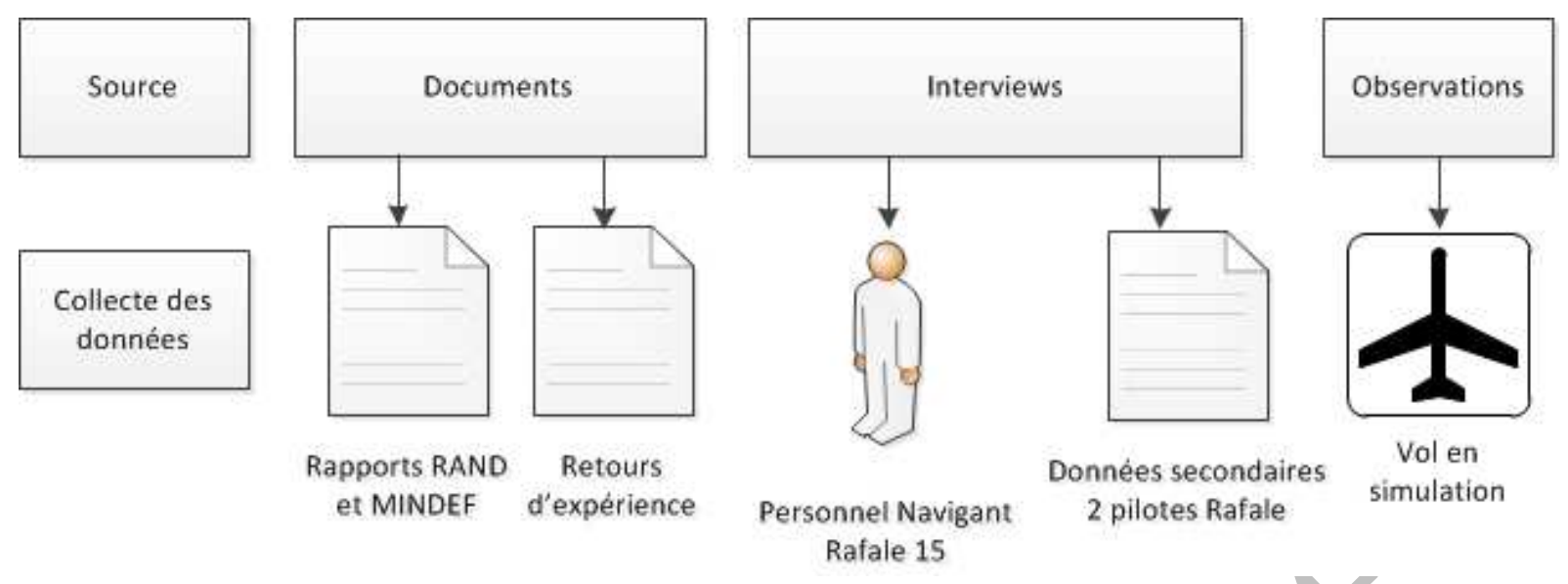

Figure 2 : La collecte de données par triangulation : illustration à partir du thème de la créativité en vol

La partie suivante expose les résultats de terrain.

\section{RÉSULTATS}

Le codage des données de terrain conduit à suggérer que Liaison 16 améliore la créativité dans le processus d'élaboration de tactiques grâce aux nouvelles possibilités qu'elle offre lors de la réalisation des missions de combat, d'une part, et à l'enrichissement des constructions collectives (scénarios tactiques élaborés au sol) lors de leur préparation, d'autre part. Les sous-sections suivantes illustrent comment cette dynamique expérimentation/conception constitue un processus de créativité.

\section{III.1 En vol, un apport de la représentation riche et de la flexibilité}

Les entretiens que nous avons menés nous ont permis de découvrir que la Liaison 16 offre aux pilotes une conscience accrue de la situation (situational awareness) durant la réalisation de leurs missions et les soulage de fastidieux calculs (Godé et Lebraty, 2013). Ce faisant, le système améliore à la fois les capacités de détection et de mise en œuvre des opportunités offertes aux pilotes en vol.

En procurant aux pilotes des informations tactiques en quasi temps réel et en opérant un lien entre ces informations, la Liaison 16 leur procure une conscience de la situation sans précédent. Un pilote nous propose la métaphore suivante : "Imaginez qu'on vous mette dans le noir, dans un endroit que yous ne connaissez pas, on se met tous à courir et on a juste une torche pour voir ce qu'on a devant nous. Et bien la différence avec la Liaison 16, c'est comme si vous mettez la lumière dans la pièce avant de partir courir. D'un coup vous voyez tout et vous comprenez quoi faire et comment faire ». Concernant les évènements inattendus de nature tactique (par exemple, la détection d'un avion ennemi, une unité commando prise au piège, etc.), les pilotes les gèrent en temps réel. Comme le précise l'un d'entre eux : « entre ce qui a été planifié et ce qu'on vit en l'air, c'est potentiellement très différent. C'est dans le moment qu'on découvre l'inattendu et il faut savoir s'adapter en temps réel ». Sans la Liaison 16, ces situations sont plus complexes à gérer car les pilotes ne bénéficient pas d'une représentation tactique aussi fine et actualisée. Un pilote de Rafale nous raconte : « La liaison de données, c'est une grosse révolution. Dans les grosses COMAO [Composite Air Operation - ensemble d'appareils ayant des capacités et des missions différentes mais devant travailler conjointement], ceux qui avaient la L16 ils disaient rien, ils n'avaient pas besoin, ils voyaient tout, pendant que les autres étaient en permanence en quête d'informations : 'request picture, request picture!'”. 
Par ailleurs, sans la Liaison 16, les pilotes décollent avec une représentation de l'environnement d'action et des problèmes qu'ils auront à traiter parfois très imprécise. Tout dépend de la quantité et de la fiabilité des renseignements récoltés en amont. Sur un théâtre tel que l'Afghanistan, où les caractéristiques physiques de terrain et la présence de combattants parmi les populations civiles rendent les conditions de collecte du renseignement particulièrement difficiles, il n'est pas rare que les pilotes décollent avec une connaissance approximative des menaces et des tactiques ennemies à venir. Un navigateur de Mirage 2000D en détachement en Afghanistan pendant plusieurs semaines explique les conditions dans lesquelles se déroulaient les missions de bombardement : "En CAS [Close Air Support ou appui feu rapproché], parfois on n'a pas l'objectif. Vous devez prendre en compte les positions des amis, les positions collatérales et l'environnement. Vous avez des drones, vous avez des FAC [Forward Air Controllers - personnels au sol désignant les cibles aux avions], $s$ 'il fait nuit ou si vous devez faire du show of force ou dans les montagnes, vous passez sous la couche pour pouvoir faire ça. Et tout ça, c'est pas planifié ». Dans ces circonstances, les pilotes consacrent une large part de leurs ressources cognitives au repérage et à l'identification des risques ainsi qu'à l'anticipation des manœuvres ennemies, au sol ou en l'air. A ces charges mentales s'ajoutent bien sûr les tâches de pilotage et l'évolution très rapide dans les trois dimensions (pour la défense aérienne). Comme le précise un pilote de Rafale: "Ceux qui n'ont pas de Liaison 16, ils font en permanence du calcul mental dans leur tête. Il faut bien le comprendre. Ils calculent, calculent... et ça prend du temps!».

Avec la Liaison 16, la charge cognitive dédiée au traitement de l'information est sensiblement allégée : «avec la L16, vous pouvez donner beaucoup de vos capacités intellectuelles, vous êtes beaucoup moins saturés ». La représentation visuelle proposée sur les écrans soulage les pilotes de fastidieux calculs : les symboles et les couleurs sont non ambigus et facilitent la compréhension de la situation : un leader explique : "par exemple, je vais voir les symboles et je vais vite savoir si mes équipiers target [suivent et pointent] bien les bonnes cibles ». Par ailleurs, le fait que les relations entre les données de terrain évoluent en temps réel épargne aux pilotes une construction mentale laborieuse de l'environnement d'action. Un pilote précise : «avec la Liaison 16, vous n'avez plus à vous construire une représentation mentale de la situation tactique. Le système le fait pour vous! Du coup, vous pouvez donner beaucoup de vos capacités intellectuelles sur le plan tactique, vous êtes beaucoup moins saturés ». Le Mission Commander sur Awacs insiste: "Avec le Rafale et la Liaison 16, les tâches de gestion de l'information ont considérablement diminué, certaines d'entre elles étant maintenant réalisées par le système. Ce faisant les équipages AWACS peuvent davantage se concentrer sur les missions mêmes de contrôle de l'espace aérien ».

C'est dans ce cadre que s'exprime l'amélioration de la flexibilité supportée par le système. Celle-ci s'exprime dans l'ajustement des tactiques dans l'action au regard des caractéristiques de l'environnement. Un pilote explique : «lorsque les situations sont complexes, le jugement humain devient primordial et permet de prendre les bonnes décisions. La Liaison 16, elle nous aide en nous permettant de comprendre ce qui se passe et de nous ajuster ». Dans ce cas, les opportunités tactiques sont plus nombreuses : la chorégraphie telle qu'elle a pu être imaginée durant la phase de briefing est réajustée in situ par les pilotes et plus spécifiquement par le leader de la formation, en charge de la gestion de la mission. L'un d'entre eux explique : «on a la possibilité d'utiliser beaucoup moins la radio [...], on a la possibilité d'utiliser notre armement grâce à la L16 [...], tu peux filtrer des informations en émission/réception. On peut aussi taper des choses à la main avec le free text de la L16 [sur les Mirage], c'est-à-dire que là on peut donner des indications ou quoi que ce soit». Le temps gagné grâce au système est alloué à l'analyse de la situation et à l'ajustement des tactiques.

Pour autant, les acteurs ne sont pas dans une situation d'improvisation dans la mesure où ils 
ont envisagé un ensemble de scénarios durant la phase de préparation ainsi que les réponses appropriées à mettre en œuvre. Un pilote explique : "on ne réinvente pas la poudre. Les tactiques de base, c'est toujours la même chose. Là où on va plus loin avec le Rafale, c'est avec la liaison de données tactiques et toutes les possibilités d'affinement tactique qu'elle nous offre! ». Par ailleurs, les pilotes évoluent dans un environnement fortement réglementé et contraignant. Lorsqu'ils se trouvent sur théâtre extérieur par exemple, les règles d'engagement, déterminées par la sphère politique, stipulent précisément leur marge de manœuvre. Enfin, le réajustement se fait sous la supervision du leader, qui fait évoluer les symboles et leur couleur sur les écrans des pilotes. Un équipier décrit: "J'ai tiré sur un Bee Gees et ça l'arrange un peu [son leader]... et si ça l'arrange mais que je ne l'ai pas fait, il met un pointeur dessus ». En d'autres termes, chaque pilote est créatif selon son niveau de responsabilité (directement corrélé à son niveau de qualification) qu'il connaît et accepte sans condition. Dans ces circonstances, on observe une réorganisation synergique et harmonieuse in situ de la formation.

\section{III.2 Au sol, un enrichissement des scénarios}

La préparation de mission s'élabore principalement durant le briefing, juste avant de partir en vol. Informés des caractéristiques de terrain et des dangers potentiels, les pilotes y abordent les questionnements tactiques, la méthode de progression ainsi que les rôles de chacun. Ils s'accordent également sur les éléments de communication (mots code) et les fréquences à utiliser. Il s'agit d'organiser le déroulement de la mission, avec comme « chef d'orchestre » le leader s'assurant que chacun sache à quoi s'attendre, quoi faire et sous quelles conditions. Comme le suggèrent les données récoltées auprès des pilotes de Mirage travaillant sans Liaison 16, les situations imprévisibles ne sont évoquées que sous l'angle technique. C'est ce que les pilotes nomment les « what if », qui posent la question de savoir quelles procédures appliquer si un problème associé au fonctionnement dú système survient en vol, tel qu'une panne moteur ou la perte de la L16 par exemple.

Avec la Liaison 16, grâce à l'obtention d'une représentation précise et actualisée de la situation tactique, l'anticipation de l'inattendu lors de la phase de préparation de la mission devient possible, en particulier à partir de la mise en place de scénarios de plus en plus sophistiqués. Un pilote illustre la situation suivante : "le problème avec le combat aérien, c'est si on voit les avions ennemis, eux ils vous voient aussi. Avec la Liaison 16, on n'a pas toujours besoin de visuel. On peut envoyer un avion qui attire la cible pendant que l'autre, qui a tout éteint et devient indétectable, l'attend en embuscade. Ces nouvelles possibilités, c'est sûr qu'on les prend en compte pendant le briefing! ». Ainsi, le leader peut se permettre d'imaginer des situations critiques et complexes (ennemis plus nombreux que prévu au départ, systèmes de défense au sol défaillants, etc.) et de préparer ses pilotes à y faire face en envisageant les éléments de réponse adéquats. Les personnels se projettent dans ces situations d'autant plus facilement que les risques de tir fratricide, de collision, etc., s'ils sont toujours discutés au briefing, sont sensiblement réduits : "La Liaison 16 permet d'obtenir un meilleur partage de la situation tactique contribuant ainsi à limiter les risques d'erreur de tir, de dommages collatéraux et de tirs fratricides". Ainsi, les pilotes ne sont plus obligés de se cantonner aux «what if » dans la mesure où ils savent pouvoir compter sur un système qui leur fournira des informations fiables et appropriées pour gérer la criticité des situations. Un pilote insiste : "dans l'orientation de ce qu'on peut faire, la L16 ouvre beaucoup de nouvelles portes! Elle a révolutionné la manière dont on utilise un avion de combat». Un autre souligne : "la Liaison 16 nous offre de nouvelles opportunités sur les tactiques aériennes à mettre en ouvre ». Il continue : "elle impacte le " game plan » c'est-à-dire la façon dont on va "jouer » la mission en fonction de l'évolution de la situation. Et on a plein de nouvelles possibilités avec la L16. Par exemple, si j'éteins mon radar, mon ennemi ne me détecte plus. 
Je deviens très discret. Et moi, je vois tout ce qui se passe à 360 degrés. Je peux arriver par derrière par exemple. En fait, ça ouvre des portes énormes, et on en est qu'au début! ».

En envisageant des scénarios tactiques originaux, les pilotes exacerbent ainsi leur vigilance en amont de la mission. Ils construisent mentalement la façon dont les scénarios envisagés seront visuellement présentés par le système (symboles génériques présentant une position, couleur, etc.). Un pilote explique : "moi, avec la Liaison 16, je sais que je vais avoir une vision globale mais ce qui est très important c'est que je vais construire un schéma mental de ce que nous allons faire et de ce que l'autre [l'ennemi] va faire ». Ce faisant, la charge de stress associée à la gestion d'événements complexes est réduite. Pour expliquer la réduction du stress, un navigateur nous décrit la situation suivante : "On part sur une mission planifiée et d'un seul coup, notre chaîne hiérarchique estime qu'on a une frappe urgente à faire sur un endroit. Une fois qu'elle a les coordonnées, elle les envoie au C2 [Commandement et Contrôle] qui nous les renvoie par la L16, et pouf! On a une cible qui va apparaître en visu!».

De même, en retour de mission, lors du debriefing, la Liaison 16 est utilisée en complément d'autres systèmes de debriefing pour restituer le déroulement de la mission. Un navigateur précise : "au débriefing, comme on a l'enregistrement de toutes nos visualisations, on peut voir exactement les temps de tir et les distances par exemple. On reporte alors toutes ces données sur les systèmes classiques de débriefing et on a une vision très claire de tout ce qui s'est passé ». Ainsi, les opportunités saisies lors de la mission, les tactiques mises en œuvre, les erreurs potentiellement commises sont analysées par les pilotes. Ces discussions viennent alimenter et enrichir le socle de connaissances communes.

La Liaison 16 offre donc à ses usagers la possibilité d'exprimer leur créativité dans la construction des scénarios de mission en améliorant la représentation des événements qui peuvent survenir une fois en vol et en élargissant le champ des réponses à mettre en œuvre pour y faire face. Dans ce cadre, l'orchestration du briefing est réalisée à partir d'une partition beaucoup plus complète et ouverte qu'auparavant.

\section{DISCUSSION}

L'étude de cas permet d'affiner notre compréhension des effets d'un système d'aide à la décision centré réseaux du type Liaison 16 sur la créativité en situation extrême.

Nous pouvons ainsi considérer qu'une mission en escadron est une construction collective, pouvant être plus ou moins créative, selon le contexte de son élaboration, les caractéristiques des acteurs et les contraintes de l'environnement. De plus, les situations extrêmes exigent à la fois de l'anticipation avant l'action et de la flexibilité durant l'action. Nous avons pu montrer que L16 satisfaisait à ces deux contraintes en renforçant la créativité des équipes. D'une manière générale, la raison principale nous conduisant à cette constatation réside dans la structure centrée-réseau du SAD. En effet, en offrant une vision d'ensemble des parties prenantes d'une situation et des liens les régissant, le décideur expert peut bâtir une représentation schématique de cette situation. Ce gain en abstraction conduit le décideur à trouver rapidement le nombre restreint de règles d'actions classiques applicables et ainsi lui permet d'envisager des schèmes potentiellement inédits. Ces schèmes constituent alors le fondement de décisions créatives.

\section{IV.1 L'intégration de la Liaison 16 dans les dimensions de la créativité}

En nous reportant à notre grille de lecture de la créativité, nous sommes en mesure d'affiner le rôle joué par le système d'aide à la décision sur les quatre dimensions (personne/groupe, processus, produit/service et environnement) liées à la créativité : 
(1) L'acteur : le système permet un allègement de la charge cognitive de l'acteur évoluant en situation extrême. De ce fait, ce dernier a le temps et des ressources cognitives disponibles pour imaginer de nouvelles voies dans le cadre prédéfini de sa mission. Pour revenir au terrain, les caractéristiques des pilotes ont un impact fort. En effet, ces derniers ont un haut niveau de compétence doublé d'un certain goût pour le dépassement des limites. Sans ces caractéristiques, nous estimons que la charge de travail, qui n'est pas gérée par le système et que les pilotes doivent traiter, serait encore trop lourde pour leur laisser la possibilité de décider de façon créative, c'est-àdire d'enrichir les tactiques préalablement décidées et pas seulement de réagir en situation d'incertitude.

(2) Le processus : la Liaison 16, en améliorant la visualisation et l'organisation des informations durant l'étape préparatoire du processus créatif, contribue à enrichir les possibilités d'action, et donc les opportunités d'élaborer des manœuvres plus créatives. Ce résultat fait écho au modèle "Geneplore » proposé par Ward et al.(1999), pour lequel la créativité comporte deux phases : une phase de génération et une phase d'exploration. Dans la phase générative, les individus construisent des représentations mentales, que les auteurs nomment des «structures pré-inventives», à partir de synthèses, d'associations d'idées et d'informations. Dans la phase exploratoire, ces représentations sont interprétées et expérimentées: leurs propriétés et leurs implications sont testées, reconfigurées, étendues ou réduites pour faire éventuellement l'objet d'une nouvelle itération. Ainsi, en offrant un nouvel espace de travail, la Liaison 16 constitue une source d'inspiration durant la phase générative : les acteurs peuvent imaginer de nouveaux processus d'action et en simuler collectivement les résultats avant même de les mettre en œuvre. De ce fait, même si durant la préparation de l'action, les usagers n'utilisent pas à proprement parler le réseau sur lequel repose le système, ils en projettent les bénéfices pour construire des processus d'action nouveaux. Durant la mission, le SAD soutient également le travail exploratoire car, en leur dégageant des capacités cognitives, il permet aux pilotes de mettre leurs représentations à l'épreuve du terrain et d'élaborer les manœuvres destinées à s'ajuster à la situation complexe. Les individus sont créatifs car ils peuvent s'appuyer sur des processus à la fois antérieurs (inspiration/simulation) et concourants à l'action (expérimentation/ajustement).

(3) La production d'un bien ou d'un service original/de valeur: un système d'aide à la décision telle que la Liaison 16 améliore sensiblement la performance de la décision à la fois au niveau individuel et collectif. En effet, en explorant l'espace de liberté créative offert par le système (processus d'action avant/après), les acteurs sont en mesure de remplir plus efficacement leur mission et donc de produire un service de qualité. L'accroissement de la capacité d'anticipation participe à cette amélioration de la qualité en favorisant une réduction des effets de surprise : en effet, le système permet aux acteurs (1) d'envisager un grand nombre de situations imprévues et (2) de libérer l'espace cognitif dont ils auront besoin pour " gérer » cette situation au mieux.

(4) L'environnement: décider en situation extrême implique de savoir articuler des contraintes d'action lourdes et obligatoires (règles, procédures, etc.) tout en sachant s'ajuster aux évolutions incessantes. Alors même que la littérature tend à considérer que les systèmes d'aide à la créativité doivent favoriser l'absence de contraintes afin de laisser libre cours à la serendipity de l'esprit, nous observons au contraire que la mise en place de nouvelles contraintes (la Liaison 16 demeure un SAD certes flexible mais les données qu'il contient suivent une structure rigide et fixée par les caractéristiques du cadre d'emploi) conduit à une redéfinition de l'espace de travail 
favorisant l'émergence de processus d'action nouveaux. La créativité des usagers en situation extrême s'exprime donc au sein d'un contexte défini à la fois par ses dimensions contraignantes et ses capacités habilitantes.

\section{IV.2 L'évidence d'un processus créatif}

A un niveau de détail plus précis, c'est le système d'itérations entre la représentation des éléments de la mission, la saisie d'opportunités et l'enrichissement des scénarios qui est à la base du processus créatif. En offrant aux équipes une visualisation des éléments du problème et des liens entre ces éléments, L16 permet :

(1) une amélioration de la compréhension de la situation et une présentation de la plupart des paramètres dont ils ont besoin pour agir efficacement, puisque le système offre des informations supplémentaires que les équipes vont pouvoir associer aux expériences et connaissances déjà détenues ;

(2) l'expérimentation de nouvelles manœuvres, l'ajustement plus fin des tactiques dans l'action et la saisie des opportunités qui se présentent, favorisant ainsi une réorganisation synergique et harmonieuse de la formation en vol. Grâce à la Liaison 16 , les pilotes disposent d'une marge de manœuvre supplémentaire par rapport au système phonique. En fonction de leur niveau de responsabilité, ils ont la possibilité d'exploiter leur potentiel en termes d'initiative tactique. Les acteurs sont rassurés par ce système qui leur procure en temps réel les informations dont ils ont besoin. Cela leur ouvre alors la possibilité de rechercher les meilleurs moyens de s'ajuster à la situation, voire d'expérimenter des scénarios qu'ils ont imaginés. Cette liberté nouvelle s'exerce bien sûr en respect des règles d'engagement et de sécurité des vols en vigueur ;

(3) l'intégration de ces nouvelles possibilités dans les tactiques et scénarios de missions et donc l'imagination de solutions plus sophistiquées et complexes que précédemment. Bien entendu, ils n'inventent pas ici des tactiques radicalement nouvelles, dans la mesure où les contraintes liées à l'avion et aux capacités humaines à encaisser les facteurs de charge restent les mêmes. En revanche, les pilotes s'ouvrent à des manœuvres jusqu'ici difficilement envisageables car trop complexes à mettre en œuvre sans Liaison 16.

En termes de créativité, le SAD joue ici le rôle de source d'inspiration, permettant aux acteurs d'aborder la situation de façon originale et d'imaginer des solutions et mouvements variés. Ainsi, même si la Liaison 16 n'a pas pour vocation originelle de favoriser l'émergence de la créativité, ce système y parvient. Ceci constitue un résultat majeur. En effet, dans notre cas, la créativité naît de deux facteurs. Premièrement, un système centré réseaux offrant à la fois une vision de l'ensemble des parties prenantes et une flexibilité de manipulation permettant à l'utilisateur d'imaginer des combinaisons inédites. Deuxièmement, l'intégration de ce système au sein d'une procédure organisationnelle globale incluant des moments d'échanges et de réflexion. Ces moments nous apparaissent indispensables pour la cristallisation des idées et donc l'émergence de la créativité. Ces constats illustrent le fait que la créativité ne se décrète pas (même à l'aide d'outils sophistiqués), mais qu'elle s'organise et nécessite un accompagnement organisationnel.

Cette recherche nous permet finalement de mettre en relief la dimension collective de la créativité, qui apparaît nettement ici. Ainsi, chaque mission peut être conçue comme une création collective originale. En faisant un parallèle avec la musique, nous constatons que les pilotes usagers de la Liaison 16 peuvent non seulement choisir parmi un grand nombre de morceaux, mais ont également la possibilité de réaliser une "improvisation » autour d'une partition donnée, tout en restant sous le contrôle du leader. Autour du SAD centré réseaux, les acteurs ayant un niveau élevé d'expertise et partageant régulièrement des expériences 
communes en milieu fortement contraint, élaborent ensemble des scénarios. Ils les « jouent» en mission, rendant ainsi leurs résultats plus performants, mieux adaptés à leur environnement et plus innovants. Ce sont d'ailleurs d'excellents joueurs. Cette excellence résulte du contexte extrême dans lequel ils sont employés. En effet, la sélection est draconienne, utilisant notamment des méthodes issues de la technique des incidents critiques (Flanagan, 1954) et s'opère de plus tout au long de la formation, ce qui favorise l'émergence d'hommes et de femmes de grande qualité. Les pilotes possèdent les compétences requises pour gérer le paradoxe entre la nécessaire rigueur dans le suivi des ordres et des règles (d'engagement, de sécurité des vols, etc.) et la capacité à s'adapter et trouver des solutions face à des situations imprévues. Un entraînement quotidien contraignant leur permet d'acquérir cette souplesse d'exécution en respect des règles. Cette flexibilité cognitive constitue aussi une condition favorisant l'émergence de créativité (Ritter et al., 2012).

\section{CONCLUSION}

L'objet de cet article était de répondre à la question de recherche suivante : « quels sont les effets d'un système d'aide à la décision de type centré réseaux sur la créativité lorsque les décideurs sont confrontés à des situations extrêmes ? ». Après avoir mené une étude exploratoire sur le comportement de pilotes de chasse utilisant un système d'aide à la décision centré réseaux nommé Liaison 16, nous mettons en lumière que ce système intégré dans une procédure organisationnelle et servi par des décideurs experts favorise la créativité. Ce résultat constitue une surprise car rien ne semblait prédisposer la Liaison 16 à faire émerger de la créativité. En ce sens, ce résultat constitue une singularité. La richesse de notre terrain nous a permis d'affiner ce lien en suggérant l'émergence d'un réel processus de créativité autour de la visualisation de la situation, la construction d'opportunités et l'enrichissement des scénarios de mission. De ce point de vue, les effets de ce type de système joueraient sur les quatre facteurs constitutifs de la créatiyité (personne/groupe, processus, produit/service et environnement). De plus, il renforcerait la dimension collective de la créativité, en élargissant la gamme des manœuvres imaginables et en facilitant l'ajustement mutuel.

A travers une analyse approfondie d'opérations militaires en situation extrême, cet article pose les bases d'un cadre théorique qui vise à proposer une prise en compte globale du processus de créativité dans une organisation utilisant massivement des technologies de l'information.

Cet article se situe dans un contexte spécifique certes, mais la tension de la situation et l'ampleur des enjeux peuvent se retrouver dans des situations managériales concrètes. Cette recherche possède ainsi un double intérêt pour les managers. Premièrement et à l'instar de Thatcher et Brown (2010) qui montrent que la communication médiée est aussi, sinon plus importante pour la créativité que la communication en face à face (p. 298), nous mettons en évidence qu'une technologie de l'information peut favoriser la créativité. Cependant, et ceci constitue une originalité forte de cette recherche, il ne s'agit pas d'un système visant directement à favoriser la créativité, mais ce sont les caractéristiques de flexibilité de l'interface et l'intégration de l'ensemble des acteurs qui favorisent cette créativité. Or, ces caractéristiques constituent le fondement des systèmes centrés réseaux. Il nous apparaît alors important de proposer le développement de tels systèmes mais appliqués à des décisions managériales. La mise à disposition de données géographiques et de logiciels pour les gérer peut constituer le fondement de tels systèmes. L'utilisation collaborative d'un logiciel comme Google Earth Pro constitue une illustration, certes basique mais potentiellement pertinente. En effet, l'ajout de règles de catégorisation des données (code couleur selon les types de données : par exemple, les emplacements des concurrents en rouge, les fournisseurs en vert et les magasins de l'entreprise en bleu) et l'utilisation du logiciel pour résoudre un problème comme le choix d'une nouvelle implantation, pourront conduire à envisager des solutions inédites. 
Deuxièmement, outre les caractéristiques du système, la créativité nécessite une intégration dans un processus organisationnel. Il peut alors apparaître paradoxal que la rigueur des procédures militaires et le sens de la précision développés dans les phases de briefing, de vol ou de debriefing soient source de créativité, mais c'est pourtant le cas. Ce type de procédure peut constituer une source d'inspiration pour des cadres opérationnels. Notre étude rappelle le fait qu'il n'y a pas de créativité sans environnement contraint comme le stipulent Bonnardel et Zenasni (2010). Nous rejoignons aussi les travaux de Wang et al. (2012) qui proposent un processus rigoureux supportant l'innovation et la créativité dans le design de nouveaux produits ou services. La transposition des systèmes électroniques de restitution de mission (la Liaison 16 le complète d'ailleurs en escadron Rafale)-au monde des affaires peut alors sembler une piste prometteuse. En retraçant avec rigueur et factuellement le déroulement de la mission, un tel système conduit les acteurs à proposer de nouvelles solutions sur la base de faits objectifs.

L'analyse de la portée de notre réflexion nous conduit cependant à en considérer les limites. Dans la mesure où cette recherche a fait l'objet d'une étude de cas unique, le nombre d'observations est trop restreint pour permettre une généralisation. Même si nos résultats offrent des éléments de réponse théoriques à la question des effets d'un système d'aide à la décision centré réseaux, ils restent fortement attachés au terrain et ne peuvent difficilement être étendus à d'autres populations ou d'autres contextes. Pour autant, ce travail ouvre au moins deux pistes de réflexion pour la recherche future. Tout d'abord, une réponse organisationnelle devrait maintenant pouvoir être envisagée autour de la Liaison 16. L'intégration organisationnelle se traduit par la formalisation de nouvelles procédures qui peuvent être vues comme des innovations. Certaines évolutions peuvent être incrémentales ou bien radicalement changer la doctrine d'emploi des forces. Dès lors, une observation de la production doctrinale de l'armée de l'air pourrait constituer un enrichissant prolongement de notre article. Ensuite, cette recherche pourrait être ré-analysée à l'aune du cadre théorique de l'appropriation (De Vaujany, 2011). Dans notre cas, il s'agirait d'une appropriation holiste (du système, des procédures et du contexte d'emploi) et il pourrait être intéressant de rechercher des régularités ou des singularités dans l'apparition de créativité. Enfin, cette recherche constitue une brique dans l'étude plus générale des phénomènes de créativité. Il conviendrait maintenant de prendre aussi en compte d'autres facteurs et notamment les synergies entre comportements amis et ennemis. En effet, la redoutable inventivité de l'ennemi dans ses contre-mesures est un facteur qui mérite d'être pris en compte.

\section{REFERENCES}

Abraham, J. \& Boone, L. W. (1994), ComputerBased Systems and Organizational Decision Making : An architecture to support Organizational Innovation. Creativity Research Journal, Vol., n ${ }^{\circ}$ 111-123.

Alter, S. (1977), A Taxonomy of Decision Support Systems. Sloan Management Review, Vol. $19, \mathrm{n}^{\circ} 1,39-57$.

Alter, S. (2004), A work system view of DSS in its fourth decade. Decision Support Systems, Vol. 38, n ${ }^{\circ}$ 3, 319-327.

Amabile, T. (1988), A model of creativity and innovation in organization. Dans Staw, B. M. \& Cummings, L. L. (Eds.) Research in Organizational Behaviour, 123-167. Chicago, Aldine Publishing.

Amabile, T. (1997), Motivating creativity in organizations : on doing what you love and loving what you do. California Management Review, Vol. 40, $\mathrm{n}^{\circ}$ 1, 39-58. 
Amabile, T., Hadley, C. \& Kramer, S. (2002), Creativity under the gun. Harvard Business Review, Vol. 80, ${ }^{\circ}, 52-61$.

Angot, J. \& Milano, P. (2007), Comment lier concepts et données ? Dans Thietart, R.-A. (Ed.) Méthodes de recherche en management 3ème Ed., 173-191. Paris, Dunod.

Applegate, L., Cash, J. \& Mills, D. (1988), Information technology and tomorrow's manager. Harvard Business Review, Vol. 66, $\mathrm{n}^{\circ}, 128-136$.

Baker, J., Jones, D. \& Burkman, J. (2009), Using Visual Representations of Data to Enhance Sensemaking in Data Exploration Tasks. Journal of the Association for Information Systems, Vol. 10, $\mathrm{n}^{\circ}$ 7, 533-559.

Barbaroux, P., Godé-Sanchez, C., Mérindol, V. \& Versailles, D. (2008), Les compétences de l'Armée de l'air à horizon 2015 : opérations aériennes et introduction du Rafale. Paris, CReA- Bureau Plans de l'Etat-major de l'Armée de l'air.

Benssam, A., Berger, J., Boukhtouta, A., Debbabi, M., Ray, S. \& Sahi, A. (2007), What middleware for network centric operations? Knowledge-Based Systems, Vol. 20, ${ }^{\circ} 3$, 255-265.

Bonnardel, N. (2000), Towards understanding and supporting creativity in design: analogies in a constrained cognitive environment. Knowledge Based Systems, Vol. 13, ${ }^{\circ}$ 7-8, 505-513.

Bonnardel, N. \& Marmèche, E. (2005), Towards supporting evocation process in creative design: a cognitive approach. International Journal of Human-Computer Studies, Vol. $63, \mathrm{n}^{\circ} 4-5,422-435$.

Bonnardel, N. \& Zenasni, F. (2010), The Impact of Technology on Creativity in Design: An Enhancement? Creativity and Innovation Management, Vol. 19, $\mathrm{n}^{\circ} 2,180-191$.

Bourgeois, L. \& Eisenhardt, K. M. (1989), Strategic decision process in high-velocity environments: Four cases in the microcomputer industry. Management Science, Vol. $34, n^{\circ} 7,816-835$.

Bouty, I., Drucker-Godard, C., Godé, C., Lièvre, P., Nizet, J. \& Pichault, F. (2011), Les pratiques de coordination en situation extrême. revue Management \& Avenir, Vol. 10, $n^{\circ} 41,346-351$.

Bouty, I., Godé, C., Drucker-Godard, C., Nizet, J., Pichault, F. \& Lièvre, P. (2012), Coordination Practices in Extreme Situations. European Management Journal, Vol. In Press, $n^{\circ}$.

Comes, T., Hiete, M., Wijngaards, S. \& Schultmann, F. (2011), Decision maps : A framework for multi-criteria decision support under severe uncertainty. Decision Support Systems, Vol. 52, n' $1,108-118$.

Cooper, R. B. (2000), Information technology development creativity : a Case Study of Attempted Radical Change. Management Information System Quaterly, Vol. 24, $\mathrm{n}^{\circ} 2$, 245-276.

Couger, J., Higgins, L. \& McIntyre, S. (1993), (Un)structured creativity in information systems organizations. MIS Quarterly, Vol. 17, $\mathrm{n}^{\circ}$ 4, 375-397.

Csikszentmihalyi, M. (1996), Creativity : flow and the psychology of discovery and invention, New-York, Harper Collins.

De Vaujany, F.-X. (2011), Revisiter l'appropriation des outils de gestion : la vision improvisationnelle de Ciborra. Dans Dominguez-Péry, C. (Ed.) Valeurs et outils de gestion - De la dynamique d'appropriation au pilotage, Chapitre 1. Hermes Science Publications.

Dubey, G. \& Moricot, C. (2005), Le Rafale ou l'objet total : sociologie d'une innovation technologique. Paris, IN-/Université de Paris 1-C2SD.

Eisenhardt, K. M. (1989), Building Theories from Case Study Research. Academy of Management Review, Vol. 14, $\mathrm{n}^{\circ}$ 4, 532-550. 
Endsley, M. R. \& Garland, D. J. (2000), Situation Awareness Analysis and Measurement, Hillsdale, Lawrence Erlbaum Associates.

Fellers, J. \& Bostrom, R. P. (1993), Application of group support systems to promote creativity in information systems organizations. Paper presented at Hawaii International Conference on System Sciences.

Flanagan, J. C. (1954), The Critical Incident Technique. Psychological Bulletin, Vol. 51, $\mathrm{n}^{\circ} 4$, 327-358.

Forgionne, J. \& Newman, J. (2007), An experiment on the effectiveness of creativity enhancing decision-making support systems. Decision Support Systems, Vol. 42, $\mathrm{n}^{\circ} 4$, 2126-2136.

Fuller, R., Murthy, U. \& Schafer, B. (2010), The effect of data model representation method on task performance. Information \& Management, Vol. 47, $\mathrm{n}^{\circ} 4,208-218$.

Galletta, D., Sampler, J. \& Teng, J. T. C. (1992), Strategies for integrating creativity principles into the system development process. Paper presented at Hawaii International Conference on System Sciences.

Girin, J. (1990), L'analyse empirique des situations de gestion. Dans Martinet, A. C. (Ed.) Epistémologies et sciences de gestion, 141-182. Paris, Economica.

Gobet, F. (2002), Recherche et reconnaissance de patterns chez les experts. Revue d'Intelligence Artificielle, Vol. 16, $\mathrm{n}^{\circ}$ 1-2, 165-190.

Godé, C. \& Barbaroux, P. (2012), Towards an architecture of organizational learning: Insights from French military aircrews. The Journal of Information and Knowledge Management Systems, Vol. 42, $\mathrm{n}^{\circ}$ 1, In Press.

Godé, C. \& Lebraty, J. F. (2013), Improving decision-making in extreme environment:The case of a military Decision Support System. International Journal of Technology and Human Interactions, Vol. 9, $\mathrm{n}^{\circ}$ 2, In Press.

Gonzales, D., Hollywood, J., Kingston, G. \& Signori, D. (2005), Network-Centric Operations Case study: Air-to-Air Combat With and Without Link 16, CA, Santa Monica, Rand Corporation.

Heaton, J. (2004), Reworking Qualitative Data, London, Sage.

Huang, Z., Chen, H., Guo, F., Xu, J. J., Wu, S. \& Chen, W.-H. (2006), Expertise visualization: An implementation and study based on cognitive fit theory. Decision Support Systems, Vol. 42, n 3, 1539-1557.

Hung, S. Y. (2003), Expert versus novice use of the executive support systems: An empirical study. Vol. 40, n 3, 177-189.

Journé, B. \& Raulet-Croset, N. (2008), Le concept de situation : contribution à l'analyse de l'activité managériale dans un contexte d'ambigüité et d'incertitude.M@n@gement, Vol. 11, $\mathrm{n}^{\circ} 1,27-55$.

Kahneman, D. \& Klein, G. (2009), Conditions for intuitive expertise: A failure to disagree. American Psychologist, Vol. 64, $\mathrm{n}^{\circ}$ 6, 515-526.

Kelder, J. C. (2005), Using someone else's data: problems, pragmatics and provisions. Forum: Qualitative Social Research, Vol. 6, $\mathrm{n}^{\circ}$ 1, Art. 39.

Klein, G. (1998), Sources of Power How People Make Decisions, Cambridge, MIT Press.

Koners, U. \& Goffin, K. (2005), Learning from new product development projects : an exploratory study. Creativity and Exploration Management, Vol. 14, n 4, 334-344.

Kristensen, T. (2004), The physical context of creativity. Creativity and Innovation Management, Vol. 13, n 2, 89-96.

Kwon, D. \& Watts, S. (2006), IT valuation in turbulent times. Journal of strategic information systems, Vol. $15, \mathrm{n}^{\circ} 4,327-354$.

Lagadec, P. (1991), La gestion des crises : outils de décision à l'usage des décideurs, Paris, McGraw-Hill. 
Lebraty, J. F. \& Lebraty, J. (2010), Décision en situation, holisme et complexité. La rationalité managériale en recherches - Mélanges en l'honneur de Jacques Rojot, 137150. Paris, ESKA.

Leonardi, P. \& Barley, S. (2008), Materiality and change: Challenges to building better theory about technology and organizing. Information and Organization, Vol. 18, $\mathrm{n}^{\circ} 3,159$ 176.

Mascitelli, R. (2000), From experience : harnessing tacit knowledge to achieve breakthrough innovation. Journal of Product Innovation Management, Vol. 17, $\mathrm{n}^{\circ} 3,179-193$.

Massetti, B. (1996), An empirical Examiniation of the Value of Creativity Support Systems on Idea Generation. Management Information System Quaterly, Vol. 20, n 1, 83-97.

McCarthy, I., Lawrence, T., Wixted, B. \& Gordon, B. (2010), A multidimensional conceptualization of environmental velocity. 35, Vol. 4, n 604-626.

Mednick, S. A. (1962), The Associative Basis of the Creative Process. Psychological Review, Vol. 69, $\mathrm{n}^{\circ} 3,220-232$.

Meyer, J. A. (1997), The acceptance of visual information in management. Information \& Management, Vol. 32, $\mathrm{n}^{\circ}$ 6, 275-285.

Orlikowski, W. (2002), Knowing in practice: Enacting a collective capability in distributed organizing. Organization Science, Vol. 13, n 3, 249-273.

Orlikowski, W. (2007), Sociomaterial practices: Exploring technology at work. Organization Studies, Vol. 28, n 9, 1435-1448.

Power, D. J. \& Sharda, R. (2007), Model-driven decision support systems: Concepts and research directions. Decision Support Systems, Vol. 43, n 3, 1044-1061.

Randel, J. M. \& Pugh, H. L. (1996), Differences in expert and novice situation awareness in naturalistic decision making. International Journal of Human-Computer Studies, Vol. $45, \mathrm{n}^{\circ} 5,579-597$.

Rhodes, M. (1961), An analysis of creativity. Phi Delta Kappan, Vol. 42, n 7, 305-310.

Rickards, T. (1987), Can computers help stimulate creativity? Training implementation from a postgraduate MBA experience. Management Learning, Vol. 18, $\mathrm{n}^{\circ} 2$, 129-139.

Ritter, S. M., Damian, R. I., Simonton, D. K., van Baaren, R. B., Strick, M., Derks, J. \& Dijksterhuis, A. (2012), Diversifying Experiences Enhance Cognitive Flexibility. Journal of Experimental Social Psychology, Vol., $\mathrm{n}^{\circ}$ To be Published.

Roux-Dufort, C. (2004), La gestion de crise, un enjeu stratégique pour les organisations 2ème Ed., Bruxelles, De Boeck Université.

Seidel, S., Muller-Wienbergen, F. \& Rosemann, M. (2010), The concept of creativity in the information systems discipline: Past, present, and prospects. Communications of the Association for Information Systems, Vol. 27, $\mathrm{n}^{\circ}$.

Shaft, T. M. \& Vessey, I. (2006), The Role of Cognitive Fit In The Relationship Between Software Comprehension And Modification. MIS Quarterly, Vol. 30, n 1, 29-55.

Simon, H. A. (1997), Administrative Behavior: A Study of Decision-Making Processes in Administrative Organizations 4th Ed., Free Press.

Sprague, R. H. \& Watson, H. J. (1996), Decision Support for Management, New Jersey, Prentice Hall.

Strauss, A. \& Corbin, J. (2008), Basics of Qualitative Research Techniques and Procedures for Developing Grounded Theory 3rd Ed., London, Sage Publication.

Sung, S. Y. \& Choi, J. N. (2012), Effects of team knowledge management on the creativity and financial performance of organizational teams. Organizational Behavior and Human Decision Processes, Vol., $\mathrm{n}^{\circ} 0$.

Thatcher, S. \& Brown, S. (2010), Individual creativity in teams: The importance of communication media mix. Decision Support Systems, Vol. 49, n 3, 290-300.

Torrance, E. P. (1974), Torrance tests of creative thinking, Lexington, MA, Personnel Press. 
Van Maanen, J., Sorensen, J. \& T., M. (2007), Introduction to special topic forum: the interplay between theory and method. Academy of Management Review, Vol. 32, $\mathrm{n}^{\circ} 4$, $1145-1154$.

Wallas, G. (1926), The art of thought, New York, Harcourt Brace.

Wang, H., Ohsawa, Y. \& Nishihara, Y. (2012), Innovation support system for creative product design based on chance discovery. Expert Systems with Applications, Vol. 39, $\mathrm{n}^{\circ}$ 5, 4890-4897.

Ward, T., Smith, S. \& Finke, R. (1999), Creative Cognition. Dans Sternberg, R. J. (Ed.) Handbook of Creativity, 189-212. Cambridge University Press.

Wierenga, B. \& van Bruggen, G. H. (1998), The dependant variable in research into the effect of creativity support systems : Quality and quantity of ideas. Management Information System Quaterly, Vol. 22, $\mathrm{n}^{\circ}$ 1, 81-87.

Wirtz, B. W., Mathieu, A. \& Schilke, O. (2007), Strategy in High-Velocity Environments. Long Range Planning, Vol. 40, $\mathrm{n}^{\circ} 3,295-313$.

Woodman, R., Sawyer, J. \& Griffin, R. (1993), Toward a Theory of Organizational Creativity. Academy of Management Review, Vol. 18, n 2, 293-321.

Yin, R. K. (2003), Case study research : design and methods 3rd Ed., Thousand Oaks, California, Sage Publications. 